\title{
A note on weak convergence of the sequential multivariate empirical process under strong mixing
}

\author{
Axel Bücher* \\ Université catholique de Louvain \&S Ruhr-Universität Bochum
}

July 16, 2018

\begin{abstract}
This article investigates weak convergence of the sequential $d$ dimensional empirical process under strong mixing. Weak convergence is established for mixing rates $\alpha_{n}=O\left(n^{-a}\right)$, where $a>1$, which slightly improves upon existing results in the literature that are based on mixing rates depending on the dimension $d$.
\end{abstract}

Keywords and Phrases: Multivariate sequential empirical processes; weak convergence; strong alpha mixing; Ottaviani's inequality.

AMS Subject Classification: 60F17, 60G10, 62G30.

\section{Introduction}

Let $\left(\boldsymbol{U}_{i}\right)_{i \in \mathbb{Z}}, \boldsymbol{U}_{i}=\left(U_{i 1}, \ldots, U_{i d}\right)$, be a strictly stationary sequence of $d$ dimensional random vectors whose marginals are standard uniform. Denote the joint cumulative distribution function of $\boldsymbol{U}_{i}$ by $C$. The corresponding empirical process is defined, for any $\boldsymbol{u}=\left(u_{1}, \ldots, u_{d}\right) \in[0,1]^{d}$, by

$$
\mathbb{D}_{n}(\boldsymbol{u})=\frac{1}{\sqrt{n}} \sum_{i=1}^{n}\left\{\mathbb{I}\left(\boldsymbol{U}_{i} \leq \boldsymbol{u}\right)-C(\boldsymbol{u})\right\}
$$

*Université catholique de Louvain, Institut de statistique, Voie du Roman Pays 20, 1348 Louvain-la-Neuve, Belgium. E-mail: axel.buecher@rub.de. 
Under various types of weak dependence conditions, the process $\mathbb{D}_{n}$ is known to converge weakly in the space of bounded functions on $[0,1]^{d}$ equipped with the supremum-norm, denoted by $\left(\ell^{\infty}\left([0,1]^{d}\right),\|\cdot\|_{\infty}\right)$, to a tight, centered Gaussian process $\mathbb{D}_{C}$ with covariance

$$
\operatorname{Cov}\left\{\mathbb{D}_{C}(\boldsymbol{u}), \mathbb{D}_{C}(\boldsymbol{v})\right\}=\sum_{i \in \mathbb{Z}} \operatorname{Cov}\left\{\mathbb{I}\left(\boldsymbol{U}_{0} \leq \boldsymbol{u}\right), \mathbb{I}\left(\boldsymbol{U}_{i} \leq \boldsymbol{v}\right)\right\}
$$

see for instance Arcones and Yu (1994) and Doukhan et al. (1995) for $\beta$ mixing, Rio (2000) for $\alpha$-mixing, Doukhan et al. (2009) for $\eta$-dependence or Durieu and Tusche (2012) for multiple mixing condtions, among others. Here and throughout, weak convergence is understood in the sense of Definition 1.3.3 of van der Vaart and Wellner (1996).

In this note, we are interested in situations in which the sequence $\left(\boldsymbol{U}_{i}\right)_{i \in \mathbb{Z}}$ satisfies strong $(\alpha-)$ mixing conditions. Let $\left(X_{i}\right)_{i \in \mathbb{Z}}$ be a sequence of Banachspace valued random variables. For $a \leq b$, where $a, b \in \mathbb{Z} \cup\{-\infty, \infty\}$, let $\mathcal{F}_{a}^{b}$ denote the $\sigma$-field generated by $\left(X_{i}\right)_{a \leq i \leq b}$. The strong mixing coefficients of the sequence $\left(X_{i}\right)_{i \in \mathbb{Z}}$ are then defined by $\alpha_{0}=1 / 2$ and

$$
\alpha_{n}=\sup _{p \in \mathbb{Z}} \sup _{A \in \mathcal{F}_{-\infty}^{p}, B \in \mathcal{F}_{p+n}^{+\infty}}|\mathbb{P}(A \cap B)-\mathbb{P}(A) \mathbb{P}(B)|, \quad n \geq 1 .
$$

The sequence $\left(X_{i}\right)_{i \in \mathbb{Z}}$ is said to be strongly mixing if $\alpha_{n} \rightarrow 0$ as $n \rightarrow \infty$.

In the following, let $\alpha_{n}$ denote the mixing coefficients of the sequence $\left(\boldsymbol{U}_{i}\right)_{i \in \mathbb{Z}}$. It has been shown by $\operatorname{Rio}(2000)$ that $\mathbb{D}_{n} \rightsquigarrow \mathbb{D}$ in $\left(\ell^{\infty}\left([0,1]^{d}\right),\|\cdot\|_{\infty}\right)$ provided that $\alpha_{n}=O\left(n^{-a}\right)$ for some $a>1$, thereby improving previous results by Yoshihara (1975) and Shao and Yu (1996).

In this note, we are interested in the slightly more general sequential empirical process

$$
\mathbb{B}_{n}(s, \boldsymbol{u})=\frac{1}{\sqrt{n}} \sum_{i=1}^{\lfloor s n\rfloor}\left\{\mathbb{I}\left(\boldsymbol{U}_{i} \leq \boldsymbol{u}\right)-C(\boldsymbol{u})\right\}
$$

where $(s, \boldsymbol{u})=\left(s, u_{1}, \ldots, u_{d}\right) \in[0,1]^{d+1}$ and $\lfloor n s\rfloor$ denotes the integer part of ns. Note that $\mathbb{D}_{n}(\boldsymbol{u})=\mathbb{B}_{n}(1, \boldsymbol{u})$. Investigating the process $\mathbb{B}_{n}$ is interesting for several reasons in mathematical statistics. For instance, the process can be used to derive nonparametric tests for change point detection in a $d$-dimensional time series, see, e.g., Inoue (2001). As a second application, suppose one is interested in constructing confidence bands for some real-valued estimator that can be written as a functional of the empirical cumulative distribution function, as for instance its integral over $\boldsymbol{u} \in[0,1]^{d}$. Then, following the self-normalizing approach developed in Shao (2010), a 
weak convergence result for $\mathbb{B}_{n}$ can used to obtain confidence bands for this estimator that do not require a tuning-parameter-dependent estimation of the asymptotic covariance.

Regarding weak convergence results for $\mathbb{B}_{n}$, it is again known for various types of weak dependence conditions that

$$
\mathbb{B}_{n} \rightsquigarrow \mathbb{B}_{C} \quad \text { in }\left(\ell^{\infty}\left([0,1]^{d+1},\|\cdot\|_{\infty}\right),\right.
$$

where $\mathbb{B}_{C}$ denotes a tight, centered Gaussian process with covariance

$$
\operatorname{Cov}\left\{\mathbb{B}_{C}(s, \boldsymbol{u}), \mathbb{B}_{C}(t, \boldsymbol{v})\right\}=(s \wedge t) \sum_{i \in \mathbb{Z}} \operatorname{Cov}\left\{\mathbb{I}\left(\boldsymbol{U}_{0} \leq \boldsymbol{u}\right), \mathbb{I}\left(\boldsymbol{U}_{i} \leq \boldsymbol{v}\right)\right\},
$$

see for instance Dedecker et al. (2013) for $\beta$-mixing or Dehling et al. (2013) for multiple mixing properties, among others. To the best of our knowledge, the best rate available in the literature for strongly mixing sequences follows from the strong approximation result established in Dhompongsa (1984): if $\alpha_{n}=O\left(n^{-b}\right)$ with $b>2+d$, then (1.1) holds. It is the purpose of the present note to improve this rate to $\alpha_{n}=O\left(n^{-a}\right)$ for any $a>1$, independently of the dimension $d$, which is the same rate as established in Rio (2000) for the non-sequential process $\mathbb{D}_{n}$. The proof of this result is inspired by the proof of Theorem 2.12.1 in van der Vaart and Wellner (1996) and is based on an adapted version of Ottaviani's inequality, see Proposition A.1.1 in the last-named reference, to strongly mixing sequences. This inequality might be of independent interest in other applications where one wants to transfer a weak convergence result from the non-sequential to the sequential setting.

\section{Main result}

Theorem 1. If $\alpha_{n}=O\left(n^{-(1+\eta)}\right)$ for some $\eta>0$, then, as $n \rightarrow \infty$,

$$
\mathbb{B}_{n} \rightsquigarrow \mathbb{B}_{C} \quad \text { in }\left(\ell^{\infty}\left([0,1]^{d+1},\|\cdot\|_{\infty}\right) .\right.
$$

For the proof of this Theorem, we need to establish weak convergence of the finite-dimensional distributions (fidis) and asymptotic tightness. Regarding weak convergence of the fidis, we can for instance apply Theorem 2.1 in Peligrad (1996). The details are omitted for the sake of brevity.

Let us consider the tightness part. For some function $f \in \ell^{\infty}\left([0,1]^{p}\right)$, $p \geq 1$, and $\delta>0$ let

$$
w_{\delta}(f)=\sup _{\|\boldsymbol{x}-\boldsymbol{y}\| \leq \delta}|f(\boldsymbol{x})-f(\boldsymbol{y})|
$$

denote the modulus of continuity of $f$. By the results in van der Vaart and Wellner (1996), Section 1.5, the following Lemma completes the proof of Theorem 1 . 
LEMma 2 (Asymptotic tightness of $\left.\mathbb{B}_{n}\right)$. Let $\alpha_{n}=O\left(n^{-(1+\eta)}\right)$ for some $\eta \in$ $(0,1)$. Then

$$
\lim _{\delta \searrow 0} \limsup _{n \rightarrow \infty} \mathbb{P}\left(w_{\delta}\left(\mathbb{B}_{n}\right)>\varepsilon\right)=0 .
$$

Proof. First, note that, by the results in Section 7 in Rio $(2000)$ and Theorem 1.5.7 and its addendum in van der Vaart and Wellner (1996), we have

$$
\lim _{\delta \searrow 0} \limsup _{n \rightarrow \infty} \mathbb{P}\left(w_{\delta}\left(\mathbb{D}_{n}\right)>\varepsilon\right)=0 .
$$

By the triangle inequality

$$
\begin{aligned}
w_{\delta}\left(\mathbb{B}_{n}\right) \leq \sup _{\left|s_{1}-s_{2}\right| \leq \delta} \sup _{\boldsymbol{u} \in[0,1]^{d}}\left|\mathbb{B}_{n}\left(s_{1}, \boldsymbol{u}\right)-\mathbb{B}_{n}\left(s_{2}, \boldsymbol{u}\right)\right| \\
+\sup _{0 \leq s \leq 1} \sup _{\|\boldsymbol{u}-\boldsymbol{v}\| \leq \delta}\left|\mathbb{B}_{n}(s, \boldsymbol{u})-\mathbb{B}_{n}(s, \boldsymbol{v})\right| .
\end{aligned}
$$

The second summand is equal to

$$
\begin{aligned}
\max _{k=1}^{n} \sup _{\|\boldsymbol{u}-\boldsymbol{v}\| \leq \delta}\left|\mathbb{B}_{n}(k / n, \boldsymbol{u})-\mathbb{B}_{n}(k / n, \boldsymbol{v})\right| \\
\quad=\max _{k=1}^{n} \sup _{\|\boldsymbol{u}-\boldsymbol{v}\| \leq \delta}\left|\sqrt{\frac{k}{n}}\left\{\mathbb{D}_{k}(\boldsymbol{u})-\mathbb{D}_{k}(\boldsymbol{v})\right\}\right|=\max _{k=1}^{n} \sqrt{\frac{k}{n}} w_{\delta}\left(\mathbb{D}_{k}\right) .
\end{aligned}
$$

Define $G_{i}(\boldsymbol{u}, \boldsymbol{v})=\mathbb{I}\left(\boldsymbol{U}_{i} \leq \boldsymbol{u}\right)-C(\boldsymbol{u})-\mathbb{I}\left(\boldsymbol{U}_{i} \leq \boldsymbol{v}\right)+C(\boldsymbol{v})$. Set $\kappa=\eta / 8$ and $\ell_{n}=\left\lfloor n^{1 / 2-\kappa}\right\rfloor$. For instance by observing that both $\mathbb{D}_{n}: \Omega \rightarrow D\left([0,1]^{d}\right)$ and $w_{\delta}: D\left([0,1]^{d}\right) \rightarrow \mathbb{R}$ are ball-measurable, we can apply Ottaviani's inequality under strong mixing, see Lemma 3, with $T=\left\{t=(\boldsymbol{u}, \boldsymbol{v}) \in[0,1]^{2 d}: \| \boldsymbol{u}-\right.$ $\boldsymbol{v} \| \leq \delta\}$ and $Y_{i}(t)=n^{-1 / 2} G_{i}(t)$, where $t=(\boldsymbol{u}, \boldsymbol{v})$. Let $\varepsilon>0$. Then we obtain

$$
\mathbb{P}\left(\max _{k=1}^{n} \sqrt{k / n} w_{\delta}\left(\mathbb{D}_{k}\right)>3 \varepsilon\right) \leq \frac{A_{n 1}+A_{n 2}+\left\lfloor n / \ell_{n}\right\rfloor \alpha_{\ell_{n}}}{1-\max _{k=1}^{n} \mathbb{P}\left(\sqrt{k / n} w_{\delta}\left(\mathbb{D}_{k}\right)>\varepsilon\right)},
$$

where $A_{n 1}=\mathbb{P}\left(w_{\delta}\left(\mathbb{D}_{n}\right)>\varepsilon\right)$ and

$$
A_{n 2}=\mathbb{P}\left(\max _{\substack{j<k \in\{1, \ldots, n\} \\ k-j \leq 2 \ell_{n}}} \sup _{\|\boldsymbol{u}-\boldsymbol{v}\| \leq \delta} \frac{1}{\sqrt{n}}\left|\sum_{i=j+1}^{k} G_{i}(\boldsymbol{u}, \boldsymbol{v})\right|>\varepsilon\right) .
$$

For sufficiently large $n$, we have $\ell_{n} \geq \frac{1}{2} n^{1 / 2-\kappa}$, whence

$$
\left\lfloor n / \ell_{n}\right\rfloor \alpha_{\ell_{n}} \leq \frac{1}{2} n^{1-(1 / 2-\kappa)-(1 / 2-\kappa)(1+\eta)}=\frac{1}{2} n^{2 \kappa-\eta / 2+\kappa \eta}=\frac{1}{2} n^{\eta / 4(\eta / 2-1)}=o(1)
$$


as $n \rightarrow \infty$. Next, $A_{n 1}$ converges to 0 as $n \rightarrow \infty$ followed by $\delta \searrow 0$ by (2.1). Moreover,

$$
\max _{\substack{j<k \in\{1, \ldots, n\} \\ k-j \leq 2 \ell_{n}}} \sup _{\|\boldsymbol{u}-\boldsymbol{v}\| \leq \delta} \frac{1}{\sqrt{n}}\left|\sum_{i=j+1}^{k} G_{i}(\boldsymbol{u}, \boldsymbol{v})\right| \leq 8 \ell_{n} / \sqrt{n} \leq 8 n^{-\kappa}=o(1),
$$

as $n \rightarrow \infty$, whence $A_{n 2}=o(1)$.

To complete the treatment of the second summand in (2.2), it remains to be shown that the denominator in $(2.3)$ is bounded away from zero for sufficiently large $n$ and small $\delta$. By (2.1), there exists $\delta_{0}>0$ such that $\limsup _{n \rightarrow \infty} \mathbb{P}\left(w_{\delta}\left(\mathbb{D}_{n}\right)>\varepsilon\right)<1 / 2$ for all $\delta \leq \delta_{0}$. Then, there exists $n_{0}=$ $n_{0}\left(\delta_{0}\right)$ such that $\mathbb{P}\left(w_{\delta_{0}}\left(\mathbb{D}_{n}\right)>\varepsilon\right)<1 / 2$ for all $n \geq n_{0}$. Therefore, for all $\delta<\delta_{0}$,

$\max _{k=n_{0}}^{n} \mathbb{P}\left(\sqrt{k / n} w_{\delta}\left(\mathbb{D}_{k}\right)>\varepsilon\right) \leq \max _{k=n_{0}}^{n} \mathbb{P}\left(w_{\delta}\left(\mathbb{D}_{k}\right)>\varepsilon\right) \leq \max _{k=n_{0}}^{n} \mathbb{P}\left(w_{\delta_{0}}\left(\mathbb{D}_{k}\right)>\varepsilon\right)<1 / 2$.

On the other hand, for $k<n_{0}$ and arbitrary $\delta>0$, we have

$$
w_{\delta}\left(\mathbb{D}_{k}\right) \leq 2 \sup _{\boldsymbol{u} \in[0,1]^{d}}\left|\mathbb{D}_{k}(\boldsymbol{u})\right| \leq 4 \sqrt{k} \leq 4 \sqrt{n}_{0}
$$

which implies that $\max _{k=1}^{n_{0}-1} \mathbb{P}\left(\sqrt{k / n} w_{\delta}\left(\mathbb{D}_{k}\right)>\varepsilon\right)=0$ for sufficiently large $n$ and all $\delta>0$. Therefore, the denominator in (2.3) is bounded from below by $1 / 2$.

Finally, consider the first suprema on the right of (2.2). It suffices to show that, for every $\varepsilon>0$,

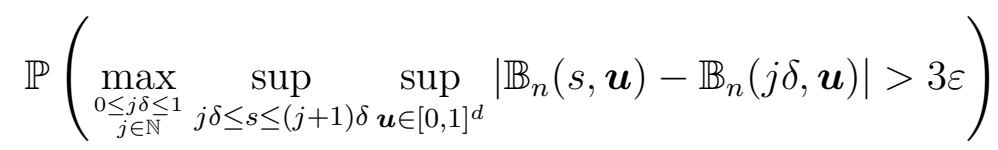

converges to 0 as $n \rightarrow \infty$ followed by $\delta \searrow 0$. By stationarity of the increments of $\mathbb{B}_{n}$ in $s$, the at most $\lceil 1 / \delta\rceil$ terms in the maximum are identically distributed. Therefore, the probability can be bounded by

$$
\begin{aligned}
& \lceil 1 / \delta\rceil \mathbb{P}\left(\sup _{0 \leq s \leq \delta} \sup _{\boldsymbol{u} \in[0,1]^{d}}\left|\mathbb{B}_{n}(s, \boldsymbol{u})\right|>3 \varepsilon\right) \\
= & \lceil 1 / \delta\rceil \mathbb{P}\left(\max _{k=1}^{\lfloor n \delta\rfloor} \sup _{\boldsymbol{u} \in[0,1]^{d}}\left|\sqrt{k / n} \mathbb{D}_{k}(\boldsymbol{u})\right|>3 \varepsilon\right) \\
\leq & \frac{\lceil 1 / \delta\rceil\left(B_{n 1}+B_{n 2}+\left\lfloor\lfloor n \delta\rfloor / \ell_{n}\right\rfloor \alpha_{\ell_{n}}\right)}{1-\max _{k=1}^{\lfloor n \delta\rfloor} \mathbb{P}\left(\sqrt{k / n} \sup _{\boldsymbol{u} \in[0,1]^{d}}\left|\mathbb{D}_{k}(\boldsymbol{u})\right|>\varepsilon\right)},
\end{aligned}
$$


by the Ottaviani-type inequality in Lemma 3, where

$$
\begin{aligned}
& B_{n 1}=\mathbb{P}\left(\sqrt{\lfloor n \delta\rfloor / n} \sup _{\boldsymbol{u} \in[0,1]^{d}}\left|\mathbb{D}_{\lfloor n \delta\rfloor}(\boldsymbol{u})\right|>\varepsilon\right) \\
& B_{n 2}=\mathbb{P}\left(\max _{\substack{j<k \in\{1, \ldots,\lfloor n \delta\rfloor\} \\
k-j \leq 2 \ell n}} \sup _{\boldsymbol{u} \in[0,1]^{d}}\left|n^{-1 / 2} \sum_{i=j+1}^{k}\left\{\mathbb{I}\left(\boldsymbol{U}_{i} \leq \boldsymbol{u}\right)-C(\boldsymbol{u})\right\}\right|>\varepsilon\right) .
\end{aligned}
$$

Here, we were allowed to apply Lemma 3 by a similar argument as before. It remains to be shown that $B_{n 1}, B_{n 2}$ and $\left\lfloor\lfloor n \delta\rfloor / \ell_{n}\right\rfloor \alpha_{\ell_{n}}$, multiplied with $1 / \delta$, converges to zero as $n \rightarrow \infty$ followed by $\delta \searrow 0$ and that the denominator in (2.4) is bounded away from zero. First of all, for $\delta \leq 1$, we have

$$
\lceil 1 / \delta\rceil\left\lfloor\left\lfloor n \delta / \ell_{n}\right\rfloor \alpha_{\ell_{n}} \leq\left(\frac{1}{\delta}+1\right) \frac{n \delta \alpha_{\ell_{n}}}{\ell_{n}} \leq 2 \frac{n \alpha_{\ell_{n}}}{\ell_{n}}=o(1)\right.
$$

as $n \rightarrow \infty$, by the same arguments as above. Second, by the PortmanteauTheorem,

$$
\begin{aligned}
\limsup _{n \rightarrow \infty} B_{n 1} & \leq \limsup _{n \rightarrow \infty} \mathbb{P}\left(\sup _{\boldsymbol{u} \in[0,1]^{d}}\left|\mathbb{D}_{n}(\boldsymbol{u})\right|>\varepsilon \delta^{-1 / 2}\right) \\
& \leq \limsup _{n \rightarrow \infty} \mathbb{P}\left(\sup _{\boldsymbol{u} \in[0,1]^{d}}\left|\mathbb{D}_{n}(\boldsymbol{u})\right| \geq \varepsilon \delta^{-1 / 2}\right) \\
& \leq \mathbb{P}\left(\sup _{\boldsymbol{u} \in[0,1]^{d}}\left|\mathbb{D}_{C}(\boldsymbol{u})\right| \geq \varepsilon \delta^{-1 / 2}\right)=\mathbb{P}\left(\sup _{\boldsymbol{u} \in[0,1]^{d}}\left|\mathbb{D}_{C}(\boldsymbol{u})\right|>\varepsilon \delta^{-1 / 2}\right)
\end{aligned}
$$

since $\sup _{\boldsymbol{u} \in[0,1]^{d}}\left|\mathbb{D}_{C}(\boldsymbol{u})\right|$ is a continuous random variable. Since additionally $\sup _{\boldsymbol{u} \in[n 11 d}\left|\mathbb{D}_{C}(\boldsymbol{u})\right|$ possesses moments of any order (cf. Proposition A.2.3 in van der Vaart and Wellner (1996)), the latter probability converges to zero faster than any power of $\delta$, as $\delta \searrow 0$.

Third, regarding $B_{n 2}$, we have

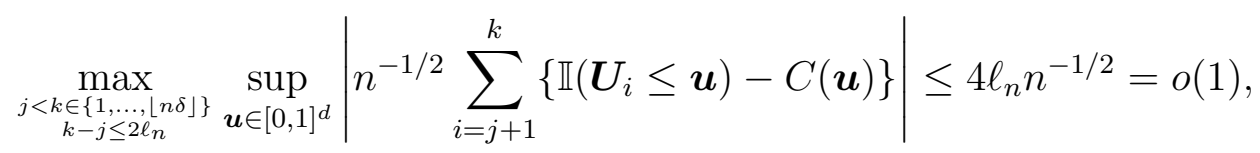

as $n \rightarrow \infty$, whence $B_{n 2}$ converges to zero as $n \rightarrow \infty$ followed by $\delta \searrow 0$ as asserted.

Finally, let us consider the denominator in (2.4). By a similar argument as before, we have from the Portmanteau Theorem that

$$
\limsup _{n \rightarrow \infty} \mathbb{P}\left(\sup _{\boldsymbol{u} \in[0,1]^{d}}\left|\mathbb{D}_{n}(\boldsymbol{u})\right|>\varepsilon\right) \leq \mathbb{P}\left(\sup _{\boldsymbol{u} \in[0,1]^{d}}\left|\mathbb{D}_{C}(\boldsymbol{u})\right|>\varepsilon\right)
$$


by continuity of $\sup _{\boldsymbol{u} \in[0,1] d}\left|\mathbb{D}_{C}(\boldsymbol{u})\right|$. Also, since $\varepsilon>0$, we obtain that $p=$ $\mathbb{P}\left(\sup _{\boldsymbol{u} \in[0,1]^{d}}\left|\mathbb{D}_{C}(\boldsymbol{u})\right|>\varepsilon\right)<1$, whence we can choose $\zeta>0$ such that $0<$ $\zeta<1-p$. It follows that there exists $n_{0} \in \mathbb{N}$ such that

$$
\sup _{k \geq n_{0}} \mathbb{P}\left(\sup _{\boldsymbol{u} \in[0,1]^{d}}\left|\mathbb{D}_{k}(\boldsymbol{u})\right|>\varepsilon\right) \leq p+\zeta
$$

Hence,

$$
\max _{k=n_{0}}^{\lfloor n \delta\rfloor} \mathbb{P}\left(\sqrt{k / n} \sup _{\boldsymbol{u} \in[0,1]^{d}}\left|\mathbb{D}_{k}(\boldsymbol{u})\right|>\varepsilon\right) \leq \max _{k=n_{0}}^{\lfloor n \delta\rfloor} \mathbb{P}\left(\sup _{\boldsymbol{u} \in[0,1]^{d}}\left|\mathbb{D}_{k}(\boldsymbol{u})\right|>\varepsilon\right) \leq p+\zeta .
$$

On the other hand, for $k<n_{0}$, we have $\sqrt{k / n} \sup _{\boldsymbol{u} \in[0,1]^{d}}\left|\mathbb{D}_{k}(\boldsymbol{u})\right| \leq \sqrt{k / n} \times$ $2 \sqrt{k} \leq 2 n_{0} / \sqrt{n}$, which implies that, for $\mathrm{n}$ large enough,

$$
\max _{k=1}^{n_{0}-1} \mathbb{P}\left(\sqrt{k / n} \sup _{\boldsymbol{u} \in[0,1]^{d}}\left|\mathbb{D}_{k}(\boldsymbol{u})\right|>\varepsilon\right)=0
$$

Hence, the denominator in (2.4) is bounded from below by $1-p-\zeta>0$ for $\mathrm{n}$ large enough. This completes the proof.

\section{An auxiliary Lemma}

Let $\left(X_{i}\right)_{i \in \mathbb{Z}}$ be a sequence of random elements in some Banach space $\mathbb{E}$. Let $T$ be some arbitrary index set and, for $i \in \mathbb{Z}$, let $G_{i} \in \ell^{\infty}(\mathbb{E} \times T)$. For $t \in T$, set $Y_{i}(t)=G_{i}\left(X_{i}, t\right)$ and $S_{n}(t)=\sum_{i=1}^{n} Y_{i}(t)$, for $n \geq 1$ and $S_{0} \equiv 0$. Finally, for $f \in \ell^{\infty}(T)$, let $\|f\|=\sup _{t \in T}|f(t)|$.

Lemma 3 (An Ottaviani-type inequality under strong mixing). Suppose that $\left\|S_{m}-S_{n}\right\|$ is measurable for each $0 \leq n<m$. Then, for each $\varepsilon>0$ and $1 \leq \ell<n$,

$$
\begin{aligned}
\mathbb{P}\left(\max _{k=1}^{n}\left\|S_{k}\right\|>3 \varepsilon\right) \times\left\{1-\max _{k=1}^{n} \mathbb{P}\left(\left\|S_{n}-S_{k}\right\|>\varepsilon\right)\right\} \\
\leq \mathbb{P}\left(\left\|S_{n}\right\|>\varepsilon\right)+\mathbb{P}\left(\max _{\substack{j<k \in\{1, \ldots, n\} \\
k-j \leq 2 \ell}}\left\|S_{k}-S_{j}\right\|>\varepsilon\right)+\lfloor n / \ell\rfloor \times \alpha_{\ell},
\end{aligned}
$$

where $\alpha_{n}$ denotes the sequence of mixing coefficients of the sequence $\left(X_{i}\right)_{i \in \mathbb{Z}}$. Proof. For $k=1, \ldots, n$, define the event $B_{k}$ by

$$
B_{k}=\left\{\left\|S_{k}\right\|>3 \varepsilon,\left\|S_{1}\right\| \leq 3 \varepsilon, \ldots,\left\|S_{k-1}\right\| \leq 3 \varepsilon\right\} .
$$


Note that these events are pairwise disjoint and that their union is given by $\left\{\max _{k=1}^{n}\left\|S_{k}\right\|>3 \varepsilon\right\}$. Furthermore, for $m=1, \ldots,\lfloor n / \ell\rfloor-1$, let

$$
C_{m}=\bigcup_{k=(m-1) \ell+1}^{m \ell} B_{k}, \quad \text { and } C_{\lfloor n / \ell\rfloor}=\bigcup_{k=(\lfloor n / \ell\rfloor-1) \ell+1}^{n} B_{k},
$$

which are also pairwise disjoint and have the same union as the $B_{k}$ 's.

Now, let us first consider a fixed $m \leq\lfloor n / \ell\rfloor-1$. Then

$$
\begin{aligned}
& \mathbb{P}\left(C_{m}\right) \times \min _{k=1}^{n} \mathbb{P}\left(\left\|S_{n}-S_{k}\right\| \leq \varepsilon\right) \\
\leq & \mathbb{P}\left(C_{m}\right) \mathbb{P}\left(\left\|S_{n}-S_{(m+1) \ell}\right\| \leq \varepsilon\right) \\
\leq & \mathbb{P}\left(C_{m},\left\|S_{n}-S_{(m+1) \ell}\right\| \leq \varepsilon\right)+\alpha_{\ell} \\
\leq & \mathbb{P}\left(C_{m}, \max _{k=(m-1) \ell+1}\left\|S_{k}\right\|>3 \varepsilon,\left\|S_{n}-S_{(m+1) \ell}\right\| \leq \varepsilon\right)+\alpha_{\ell} .
\end{aligned}
$$

Since $\left\|S_{k}\right\| \leq\left\|S_{k}-S_{(m+1) \ell}\right\|+\left\|S_{(m+1) \ell}-S_{n}\right\|+\left\|S_{n}\right\|$ for any $k=1, \ldots, n$, we have

$$
\begin{aligned}
\left\|S_{n}\right\| & \geq \max _{k=(m-1) \ell+1}^{\operatorname{me}}\left\{\left\|S_{k}\right\|-\left\|S_{(m+1) \ell}-S_{k}\right\|-\left\|S_{(m+1) \ell}-S_{n}\right\|\right\} \\
& \geq\left\{\max _{k=(m-1) \ell+1}\left\|S_{k}\right\|\right\}-\left\{\max _{k=(m-1) \ell+1}\left\|S_{(m+1) \ell}-S_{k}\right\|\right\}-\left\|S_{(m+1) \ell}-S_{n}\right\| .
\end{aligned}
$$

Therefore, we can estimate the right-hand side of (3.1) by

$$
\begin{aligned}
& \leq \mathbb{P}\left(C_{m},\left\|S_{n}\right\|>2 \varepsilon-\max _{k=(m-1) \ell+1}^{m \ell}\left\|S_{(m+1) \ell}-S_{k}\right\|\right)+\alpha_{\ell} \\
& \leq \mathbb{P}\left(C_{m},\left\|S_{n}\right\|>\varepsilon\right)+\mathbb{P}\left(C_{m}, \max _{k=(m-1) \ell+1}^{m \ell}\left\|S_{(m+1) \ell}-S_{k}\right\|>\varepsilon\right)+\alpha_{\ell} \\
& \leq \mathbb{P}\left(C_{m},\left\|S_{n}\right\|>\varepsilon\right)+\mathbb{P}\left(C_{m}, \max _{\substack{j<k \in\{1, \ldots, n\} \\
k-j \leq 2 \ell}}\left\|S_{k}-S_{j}\right\|>\varepsilon\right)+\alpha_{\ell} .
\end{aligned}
$$

Next, let us consider the case $m=\lfloor n / \ell\rfloor$. Then

$$
\begin{aligned}
& \mathbb{P}\left(C_{\lfloor n / \ell\rfloor}\right) \times \min _{k=1}^{n} \mathbb{P}\left(\left\|S_{n}-S_{k}\right\| \leq \varepsilon\right) \\
\leq & \mathbb{P}\left(C_{\lfloor n / \ell\rfloor}, \max _{k=(\lfloor n / \ell\rfloor-1) \ell+1}^{n}\left\|S_{k}\right\|>3 \varepsilon\right) \\
\leq & \mathbb{P}\left(C_{\lfloor n / \ell\rfloor},\left\|S_{n}\right\|>3 \varepsilon-\max _{k=(\lfloor n / \ell\rfloor-1) \ell+1}^{n}\left\|S_{n}-S_{k}\right\|\right)
\end{aligned}
$$




$$
\begin{aligned}
& \leq \mathbb{P}\left(C_{\lfloor n / \ell\rfloor},\left\|S_{n}\right\|>2 \varepsilon\right)+\mathbb{P}\left(C_{\lfloor n / \ell\rfloor}, \max _{k=(\lfloor n / \ell\rfloor-1) \ell+1}^{n}\left\|S_{n}-S_{k}\right\|>\varepsilon\right) \\
& \leq \mathbb{P}\left(C_{\lfloor n / \ell\rfloor},\left\|S_{n}\right\|>\varepsilon\right)+\mathbb{P}\left(C_{\lfloor n / \ell\rfloor}, \max _{\substack{j<k \in\{1, \ldots, n\} \\
k-j \leq 2 \ell}}\left\|S_{k}-S_{j}\right\|>\varepsilon\right) .
\end{aligned}
$$

Joining both cases, we have, for any $m=1, \ldots,\lfloor n / \ell\rfloor$,

$$
\begin{aligned}
\mathbb{P}\left(C_{m}\right) \times \min _{k=1}^{n} \mathbb{P}\left(\left\|S_{n}-S_{k}\right\| \leq \varepsilon\right) \\
\quad \leq \mathbb{P}\left(C_{m},\left\|S_{n}\right\|>\varepsilon\right)+\mathbb{P}\left(C_{m}, \max _{\substack{j<k \in\{1, \ldots, n\} \\
k-j \leq 2 \ell}}\left\|S_{k}-S_{j}\right\|>\varepsilon\right)+\alpha_{\ell} .
\end{aligned}
$$

Summation over $m$ finally yields the assertion.

Acknowledgements. The author is thankful to Ivan Kojadinovic for thorough proofreading and numerous suggestions concerning this manuscript.

This work has been supported in parts by the Collaborative Research Center "Statistical modeling of nonlinear dynamic processes" (SFB 823) of the German Research Foundation (DFG) and by the IAP research network Grant P7/06 of the Belgian government (Belgian Science Policy), which is gratefully acknowledged.

\section{REFERENCES}

Arcones, M. A. and B. Yu (1994). Central limit theorems for empirical and $U$-processes of stationary mixing sequences. J. Theoret. Probab. 7(1), 4771.

Dedecker, J., F. Merlevède, and E. Rio (2013). Strong approximation of the empirical distribution function for absolutely regular sequences in $\mathbf{R}^{d}$. http://hal.archives-ouvertes.fr/hal-00798305. working paper.

Dehling, H., O. Durieu, and M. Tusche (2013). A sequential empirical CLT for multiple mixing processes with application to $\mathcal{B}$-geometrically ergodic markov chains. arXiv:1303.4537.

Dhompongsa, S. (1984). A note of the almost sure approximation of the empirical process of weakly dependent random vectors. Yokohama Mathematical Journal 32, 113-121.

Doukhan, P., J.-D. Fermanian, and G. Lang (2009). An empirical central limit theorem with applications to copulas under weak dependence. Stat. Inference Stoch. Process. 12(1), 65-87. 
Doukhan, P., P. Massart, and E. Rio (1995). Invariance principles for absolutely regular empirical processes. Ann. Inst. H. Poincaré Probab. Statist. 31(2), 393-427.

Durieu, O. and M. Tusche (2012). An empirical process central limit theorem for multidimensional dependent data. Journal of Theoretical Probability, $1-29$.

Inoue, A. (2001). Testing for distributional change in time series. Econometric Theory 17(1), 156-187.

Peligrad, M. (1996). On the asymptotic normality of sequences of weak dependent random variables. J. Theoret. Probab. 9(3), 703-715.

Rio, E. (2000). Théorie asymptotique des processus aléatoires faiblement dépendants. Berlin Heidelberg: Springer.

Shao, Q.-M. and H. Yu (1996). Weak convergence for weighted empirical processes of dependent sequences. Ann. Probab. 24 (4), 2098-2127.

Shao, X. (2010). A self-normalized approach to confidence interval construction in time series. J. R. Stat. Soc. Ser. B Stat. Methodol. 72(3), 343-366.

van der Vaart, A. and J. Wellner (1996). Weak convergence and empirical processes. New York: Springer.

Yoshihara, K.-i. (1975). Weak convergence of multidimensional empirical processes for strong mixing sequences of stochastic vectors. Z. Wahrscheinlichkeitstheorie und Verw. Gebiete 33(2), 133-137. 